\title{
Pell-Lucas Collocation Method for Solving High-Order Functional Differential Equations with Hybrid Delays
}

\author{
Melike Şahin*, Mehmet Sezer \\ Department of Mathematics, Faculty of Science and Arts , Manisa Celal Bayar University, Campus of Şehit Prof. \\ Dr. İlhan Varank, 45140, Yunusemre, Manisa \\ "melike_sahin_92@hotmail.com, mehmet.sezer@cbu.edu.tr
}

Received: 20 April 2017

Accepted: 13 June 2018

DOI: $10.18466 /$ cbayarfbe. 307282

\begin{abstract}
In this study, the Pell-Lucas collocation method has been presented to solve high-order linear functional differential equations with hybrid delays under mixed conditions. The proposed method is based on the matrix forms of Pell-Lucas polynomials and their derivatives, along with the collocation points. The used technique reduces the problem to a matrix equation corresponding to a set of algebraic equations with the unknown Pell-Lucas coefficients. In addition, an error analysis based on residual function is performed and some numerical examples are presented to show the efficiency and accuracy of the method.

Keywords: Pell-Lucas polynomials, collocation method, functional differential-equations, residual error analysis, matrix method.
\end{abstract}

\section{Introduction}

In this study, we consider the high-order linear functional-differential equations with variable coefficients and mixed delays in the generalized form [1-12]

$$
\begin{aligned}
\sum_{k=0}^{m} P_{k}(x) y^{(k)}(x) & +\sum_{r=0}^{m_{1}} \sum_{s=0}^{m_{2}} F_{r s}(x) y^{(r)}\left(\alpha_{r s}+\beta_{r s}\right) \\
& =g(x), m \geq m_{1}
\end{aligned}
$$

under the conditions

$$
\begin{gathered}
\sum_{k=0}^{m-1}\left(a_{k j} y^{(k)}(a)+b_{k j} y^{(k)}(b)\right)=\lambda_{j}, \\
j=0,1,2, \ldots, m-1 .
\end{gathered}
$$

where the functions $P_{k}(x), F_{r s}$ and $g(x)$ are known function having $\mathrm{m}$-th derivatives on interval $[\mathrm{a}, \mathrm{b}]$; the constants $\alpha_{r s}, b_{k j}, \quad a_{k j}, \beta_{r s}$ and $\lambda_{j}$ are appropriate constants.

In the context of the modeling of dynamical systems, the functional differential equations in the form (1.1) play a central role in various fields such as biology, economy, electrodynamics, potential theory, electrostatics, astronomy, chemistry, mechanics, physics, etc.[1,4,9,11,12]. Most of these equations have not analytical solution and so, numerical methods may be required to obtain their approximate solutions. For example, some functional differential equations have been solved by using the numerical methods such as one-Leg $\theta$ method [9], the spline function method [2], Legendre-Gauss collocation method [4], Chebyshev operational matrix method [5], Optimal residual method [10], Dickson Collocation Method Based on Residual
Error [11], Jacobian elliptic function method [12], Chebyshev Collocation method with residual correction [13], Legendre spectral collocation method [14] and Lagrange-collocation method [15].

In recent years, some matrix and collocation methods to solve linear and nonlinear differential, integral, integrodifferential, integro-differential-difference and pantograph equations have been presented in many articles by Sezer and Coworkers [7, 8, 11, 16-18]. The purpose in this paper, by means of the above mentioned methods, is to develop a new collocation method based on Pell-Lucas polynomials and to find the approximate solution of the problem (1.1)-(1.2) as the truncated PellLucas series defined by

$$
\begin{aligned}
& y(x) \cong y_{N}(x)=\sum_{n=o}^{N} a_{n} Q_{n}(x), N \geq m, \\
& , a \leq x \leq b
\end{aligned}
$$

where $Q_{n}(x), n=0,1, \ldots, N$, denote the Pell-Lucas polynomials $[19,20] ; a_{n}, n=0,1, \ldots, N$, are unknown Pell-Lucas coefficients and $\mathrm{N}$ is any positive integer chosen such that $N \geq m$. Besides, the collocation points are defined by

and

$$
x_{i}=a+\frac{b-a}{N} i, \quad(\text { standard })
$$

$$
=\frac{b+a}{2} \frac{x_{i}-a}{2} \cos \left(\frac{\pi i}{N}\right)(\text { Cheb. Lobatto })
$$


2. Materials and Methods

\subsection{Some Important Properties of the Pell-Lucas}

\section{Polynomials [19,20]}

Pell-Lucas defined the set of polynomials $\left\{Q_{n}(x)\right\}$. The polynomials $\left\{Q_{n}(x)\right\}$ are recursively defined by the following relationships:

$$
Q_{n}(x)=2 x Q_{n-1}(x)+Q_{n-2}(x), n \geq 2
$$

$$
\text { with } Q_{0}(x)=2 \text { and } Q_{1}(x)=2 x \text {. }
$$

The Pell-Lucas polynomials $Q_{n}(x)$ can also be given explicitly by

$$
Q(x)=\sum_{k=0}^{[n / 2]} 2^{n-2 k} \frac{n}{n-k}\left(\begin{array}{c}
n-k \\
k
\end{array}\right) x^{n-2 k}
$$

The first four Pell-Lucas polynomial $Q_{n}(x)$ :

$$
\begin{gathered}
Q_{0}(x)=2, \quad Q_{1}(x)=2 x, \quad Q_{2}(x)=4 x+2, \\
Q_{3}(x)=8 x^{3}+6 x, \ldots
\end{gathered}
$$

\subsection{Fundamental Matrix Relations and Pell-}

\section{Lucas Collocation Method}

Firstly, we approximate the solution (1.3) of the problem (1.1)-(1.2) by the matrix form

$$
y(x) \cong y_{N}(x)=\boldsymbol{Q}(x) \boldsymbol{A}
$$

where

$$
\begin{gathered}
\boldsymbol{Q}(x)=\left[\begin{array}{llll}
Q_{0}(x) & Q_{1}(x) & \ldots & Q_{N}(x)
\end{array}\right] \\
\boldsymbol{A}=\left[\begin{array}{llll}
a_{0} & a_{1} & \cdots & a_{N}
\end{array}\right]^{T} .
\end{gathered}
$$

Now we clearly write the matrix form $Q_{n}(x)$, by using the Pell-Lucas polynomials $Q_{n}(x)$ given by (2.1) or (2.2), as

$$
\boldsymbol{Q}(x)=\boldsymbol{X}(x) \boldsymbol{M}
$$

\begin{tabular}{|c|c|c|c|c|c|c|c|c|c|}
\hline 2 & 0 & 0 & & 0 & 0 & & 0 & $\ldots$ & 0 \\
\hline 0 & $2^{1} \frac{1}{1}\left(\begin{array}{l}1 \\
0\end{array}\right)$ & 0 & & 0 & 0 & & 0 & $\ldots$ & 0 \\
\hline $2^{0} \frac{2}{1}\left(\begin{array}{l}1 \\
1\end{array}\right)$ & 0 & $2^{2} \frac{2}{2}$ & & 0 & 0 & & 0 & $\ldots$ & 0 \\
\hline 0 & $2{ }^{3} \frac{3}{2}\left(\begin{array}{l}1 \\
1\end{array}\right)$ & 0 & & $2^{3} \frac{3}{3}\left(\begin{array}{l}3 \\
0\end{array}\right)$ & 0 & & 0 & $\ldots$ & 0 \\
\hline $2^{0} \frac{4}{2}\left(\begin{array}{l}2 \\
2\end{array}\right)$ & 0 & $2^{2} \frac{4}{3}$ & & 0 & $2^{4} \frac{4}{4}$ & & 0 & $\ldots$ & 0 \\
\hline 0 & $2^{2} \frac{5}{3}\left(\begin{array}{l}3 \\
2\end{array}\right)$ & 0 & & $2^{1} \frac{5}{4}\left(\begin{array}{l}4 \\
1\end{array}\right)$ & 0 & & $2^{3} \frac{5}{5}\left(\begin{array}{l}5 \\
0\end{array}\right)$ & $\ldots$ & 0 \\
\hline$\vdots$ & $\vdots$ & $\vdots$ & & $\vdots$ & $\vdots$ & & $\vdots$ & $\because$ & $\vdots$ \\
\hline$N$ & & & $(N+2)$ & & & $(N+4)$ & & & \\
\hline $2^{0} \frac{N}{N} \mid \begin{array}{c}2 \\
N\end{array}$ & 0 & $2^{2} \frac{N}{N+2}$ & $\begin{array}{c}2 \\
N-2\end{array}$ & 0 & $2^{2} \frac{N}{N+2}$ & $\begin{array}{c}2 \\
N-4\end{array}$ & 0 & $\ldots$ & $\left.2^{N} \frac{N}{N}\right|_{0} ^{N}$ \\
\hline$\overline{2}\left(\frac{1}{2}\right.$ & & 2 & $\left(\frac{\pi}{2}\right)$ & & 2 & 2 & & & \\
\hline
\end{tabular}

where

$$
X(x)=\left[\begin{array}{lllll}
1 & x & x^{2} & \ldots & x^{N}
\end{array}\right] .
$$

If $\mathrm{N}$ is odd,

$$
\boldsymbol{M}^{T}=
$$

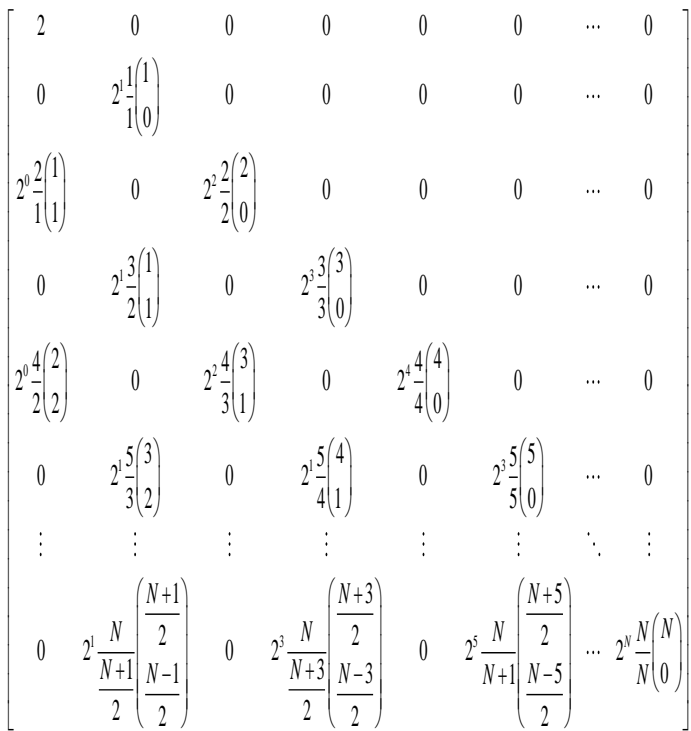

If $\mathrm{N}$ is even,

$\boldsymbol{M}^{T}=$

From the relations (2.3) and (2.4), we obtain the matrix form

$$
y_{N}(x)=\boldsymbol{X}(x) \boldsymbol{M} \boldsymbol{A} \text {. }
$$

Also, the relations between the matrix $\boldsymbol{X}(x)$ and its derivative $\boldsymbol{X}^{(k)}(x)$ are

so that

$$
\boldsymbol{X}^{(k)}(x)=\boldsymbol{X}(x) \boldsymbol{B}^{k}, \quad k=0,1, \ldots
$$

$$
B^{0}=\left[\begin{array}{ccccc}
1 & 0 & 0 & \cdots & 0 \\
0 & 1 & 0 & \cdots & 0 \\
0 & 0 & 1 & \cdots & 0 \\
\vdots & \vdots & \vdots & \ddots & \vdots \\
0 & 0 & 0 & \cdots & 1
\end{array}\right], \quad B=\left[\begin{array}{ccccc}
0 & 1 & 0 & \cdots & 0 \\
0 & 0 & 2 & \cdots & 0 \\
\vdots & \vdots & \vdots & \ddots & \vdots \\
0 & 0 & 0 & \cdots & N \\
0 & 0 & 0 & \cdots & 0
\end{array}\right] .
$$

By using the matrices (2.5) and (2.6), we have the matrix relation 


$$
\begin{gathered}
y^{(k)}(x) \cong y_{N}^{(k)}(x)=\boldsymbol{Q}^{(k)}(x) \boldsymbol{A}=\boldsymbol{X}^{(k)}(x) \boldsymbol{M} \boldsymbol{A}= \\
\boldsymbol{X}(x) \boldsymbol{B}^{k} \boldsymbol{M} \boldsymbol{A}, \quad k=0,1,2, \ldots, m
\end{gathered}
$$

and by substituting $\quad x \rightarrow \alpha_{r s} x+\beta_{r s}$ and $k \rightarrow$ $r$ in (2.7), the matrix relation

$$
\begin{aligned}
& y^{(r)}\left(\alpha_{r s} x+\beta_{r s}\right)=\boldsymbol{X}\left(\alpha_{r s} x+\beta_{r s}\right) \boldsymbol{B}^{r} \boldsymbol{M} \boldsymbol{A} \\
= & \boldsymbol{X}(x) \boldsymbol{B}\left(\alpha_{r s}, \beta_{r s}\right) \boldsymbol{B}^{r} \boldsymbol{M} \boldsymbol{A}
\end{aligned}
$$

where

$$
\begin{aligned}
& \boldsymbol{B}\left(\boldsymbol{\alpha}_{r s}, \boldsymbol{\beta}_{r s}\right)=
\end{aligned}
$$

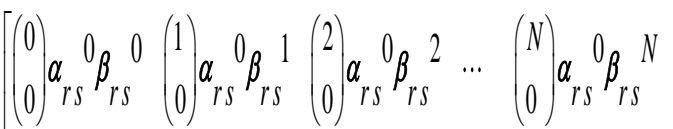

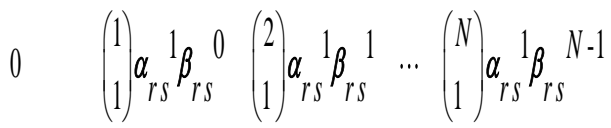

$$
\begin{aligned}
& 0 \quad 0 \quad\left(\begin{array}{l}
2 \\
2
\end{array} \alpha_{r s}{ }_{r s}^{2} \beta_{r s}^{0} \ldots\left(\begin{array}{l}
N \\
2
\end{array}\right) \alpha_{r s}^{2} \beta_{r s}^{N-2}\right. \\
& 0 \quad 0 \quad 0 \quad \ldots\left(\begin{array}{l}
N \\
N
\end{array}\right) a_{r s} N_{r s} 0
\end{aligned}
$$

Note that the matrix $\boldsymbol{X}\left(\alpha_{r s} x+\beta_{r s}\right)$ can be written as

$$
\boldsymbol{X}\left(\alpha_{r s} x+\beta_{r s}\right)=\boldsymbol{X}(x) \boldsymbol{B}(\alpha, \beta) .
$$

By substituting the relations (2.7) and (2.8) into Eq.(1.1),we obtain the matrix equation

$$
\begin{aligned}
& \left\{\sum_{k=0}^{m} P_{k}(x) X(x) B^{k}\right. \\
& \left.+\sum_{r=0}^{m_{1}} \sum_{s=0}^{m_{2}} F_{r s}(x) X(x) B\left(\alpha_{r s}, \beta_{r s}\right) B^{r}\right\} M A=g(x)
\end{aligned}
$$

and then, by placing the collocation points (1.4),the system of the matrix equations

$$
\begin{aligned}
& \left\{\sum_{k=0}^{m} P_{k}\left(x_{i}\right) \boldsymbol{X}\left(x_{i}\right) \boldsymbol{B}^{\boldsymbol{k}}\right. \\
& \left.+\sum_{\substack{m_{1}=0 \\
m_{1}=0}}^{m_{2}} F_{r s}\left(x_{i}\right) \boldsymbol{X}\left(\boldsymbol{x}_{\boldsymbol{i}}\right) \boldsymbol{B}\left(\alpha_{r s}, \beta_{r s}\right) \boldsymbol{B}^{r}\right\} \boldsymbol{M} \boldsymbol{A}=g\left(x_{i}\right) \\
& i=0,1, \ldots, N .
\end{aligned}
$$

The compact form of this system can be written as

$$
\begin{gathered}
\left\{\sum_{k=0}^{m} \boldsymbol{P}_{\boldsymbol{k}} \boldsymbol{X} \boldsymbol{B}^{\boldsymbol{k}}+\sum_{r=0}^{m_{1}} \sum_{s=0}^{m_{2}} \boldsymbol{F}_{r s} \boldsymbol{X} \boldsymbol{B}\left(\alpha_{r s}, \beta_{r s}\right) \boldsymbol{B}^{r}\right\} \boldsymbol{M} \boldsymbol{A} \\
=\boldsymbol{G}
\end{gathered}
$$

where

$$
\begin{aligned}
& \boldsymbol{X}=\left[\begin{array}{c}
X\left(x_{0}\right) \\
X\left(x_{1}\right) \\
\vdots \\
X\left(x_{n}\right)
\end{array}\right]=\left[\begin{array}{ccccc}
1 & x_{0} & x_{0}^{2} & \ldots & x_{0}^{N} \\
1 & x_{1} & x_{1}^{2} & \ldots & x_{1}^{N} \\
\vdots & \vdots & \vdots & \ddots & \vdots \\
1 & x_{N} & x_{N}^{2} & \ldots & x_{N}^{N}
\end{array}\right] \\
& \boldsymbol{G}=\left[\begin{array}{c}
g\left(x_{0}\right) \\
g\left(x_{1}\right) \\
\vdots \\
g\left(x_{N}\right)
\end{array}\right] \\
& \boldsymbol{P}_{k}=\operatorname{diag}\left[P_{k}\left(x_{0}\right) \quad P_{k}\left(x_{1}\right) \quad \cdots \quad P_{k}\left(x_{N}\right)\right] \\
& \boldsymbol{F}_{r s}=\operatorname{diag}\left[F_{j}\left(x_{0}\right) \quad F_{j}\left(x_{1}\right) \quad \cdots \quad F_{j}\left(x_{N}\right)\right] .
\end{aligned}
$$

In Eq. (2.9), the general forms of the matrices $P_{k}, X, B, F_{r s}, B(\alpha, \beta), M, A$ and $G$, respectively, are $(N+1) x(N+1),(N+1) x(N+1),(N+1) x(N+$ 1), $(N+1) x(N+1),(N+1) x(N+1),(N+1) x(N+$ $1),(N+1) x 1$ and $(N+1) x 1$.

The fundamental matrix equation (2.9) can be expressed in the form

$$
\boldsymbol{W A}=\boldsymbol{G} \text { or }[\boldsymbol{W} ; \boldsymbol{G}]
$$

where

$$
\begin{aligned}
& \boldsymbol{W}=\left[\boldsymbol{w}_{p q}\right]=\left\{\sum_{k=0}^{m} \boldsymbol{P}_{k} \boldsymbol{X} \boldsymbol{B}^{k}\right. \\
& \left.+\sum_{r=0}^{m_{1}} \sum_{s=0}^{m_{2}} \boldsymbol{F}_{r s} \boldsymbol{X} \boldsymbol{B}\left(\alpha_{r s}, \beta_{r s}\right) \boldsymbol{B}^{r}\right\} \boldsymbol{M}, \\
& p, q=0,1, \ldots, N \text {. }
\end{aligned}
$$

On the other hand, by mean of the relation (2.7), we can write the matrix forms of the conditions (1.2) as

$\boldsymbol{U}_{j} \boldsymbol{A}=\boldsymbol{\lambda}_{j}$ or $\left[\boldsymbol{U}_{j}, \boldsymbol{\lambda}_{j}\right], j=0,1,2, \ldots, m-1$,

such that

$$
\begin{aligned}
\boldsymbol{U}_{j}=\left[\begin{array}{llll}
u_{j 0} & u_{j 1} & \cdots & u_{j N}
\end{array}\right] \\
=\sum_{k=0}^{m-1}\left(a_{k j} \boldsymbol{X}(a)+b_{k j} \boldsymbol{X}(b)\right)(\boldsymbol{B})^{k} \boldsymbol{M} \\
\quad j=0,1,2, \ldots, m-1 .
\end{aligned}
$$

Consequently, in order to obtain the Pell-Lucas polynomial solution of Eq. (1.1) under the condition (1.2), we replace the $\mathrm{m}$ row matrices (2.11) by any $\mathrm{m}$ rows of the augmented matrix (2.10). Thereby we obtain the new augmented matrix 


$$
[\widetilde{\boldsymbol{W}}, \widetilde{\boldsymbol{G}}] \text { or } \widetilde{\boldsymbol{W}} \boldsymbol{A}=\widetilde{\boldsymbol{G}} .
$$

If $\widetilde{W}=\operatorname{rank}[\widetilde{W}, \tilde{G}]=N+1$, then we can write $A=$ $(\widetilde{W})^{-1} \widetilde{G}$. Thus the matrix A (thereby the Pell-Lucas coefficients $\left.a_{0}, a_{1}, \ldots, a_{N}\right)$ is uniquely determined. Also, Eq. (1.1) under the conditions (1.2) has an unique solution. This solution is given by the truncated PellLucas series (1.3).

\section{Results and Discussion}

\subsection{Accuracy of Solutions and Residual Error} Estimation

In this section, we investigate the accuracy of the obtained Pell-Lucas solutions. When $y_{N}(x)$ and its derivatives are substituted in Eq. (1.1), the solved equation is required to satisfy approximately. For $x=$ $x_{r} \in[a, b], j=0,1,2, \ldots$,

$$
\begin{aligned}
& R_{N}\left(x_{j}\right)=\sum_{k=0}^{m} P_{k}\left(x_{j}\right) y_{N}^{(k)}\left(x_{j}\right) \\
& \quad+\sum_{r=0}^{m_{1}} \sum_{s=0}^{m_{2}} F_{r s}\left(x_{j}\right) y_{N}^{(r)}\left(\alpha_{r s} x_{j}+\beta_{r s}\right)-g\left(x_{j}\right) \cong 0
\end{aligned}
$$

or

$$
R_{N}\left(x_{j}\right) \leq 10^{-x_{j}}\left(, x_{j} \text { is any positive integer }\right) .
$$

If $\max 10^{-k_{j}}=10^{-k}\left(k_{j} i \mathrm{~s}\right.$ an positive integer) is determined, then the truncation limit $N$ is increased until the difference $R_{N}\left(x_{j}\right)$ at each of the points becomes smaller than the prescribed $10^{-k}[16-18]$.

On the other hand, the accuracy of the solution can be determined and the error can be estimated by means of the residual function $R_{N}(x)$ and the mean value of $\left|R_{N}(x)\right|$ on $[a, b]$, If $R_{N}(x) \rightarrow 0$ and $\mathrm{N}$ is sufficiently enough, then the error decreases. Also, by using the Mean-Value Theorem for the residual function [18], we can estimate the upper bound of the mean error, $\overline{R_{N}}$ : $\left|\int_{a}^{b} R_{N}(x) d x\right| \leq \int_{a}^{b}\left|R_{N}(x)\right| d x$

and

$\int_{a}^{b} R_{N}(x) d x=(b-a) R_{N}(c), \quad \mathrm{a} \leq \mathrm{c} \leq \mathrm{b}$

$\Rightarrow\left|\int_{a}^{b} R_{N}(x) d x\right|=(b-a)\left|R_{N}(c)\right|$

$\Rightarrow(b-a)\left|R_{N}(c)\right| \leq \int_{a}^{b}\left|R_{N}(x)\right| d x$

$\Rightarrow\left|R_{N}(c)\right| \leq \frac{\int_{a}^{b}\left|R_{N}(x)\right| d x}{b-a}=\bar{R}_{N}$

\subsection{Numerical Examples}

Example 1. Consider the second order pantograph equation

$$
y^{\prime \prime}(x)-\frac{3}{4} y(x)-y\left(\frac{x}{2}\right)=-x^{2}+2,0 \leq x \leq 1
$$

with initial conditions

$$
y(0)=0, \quad y^{\prime}(0)=0 .
$$

The exact solution of this problem is $y(x)=x^{2}$ and the coefficients in Eq. (1.1) are defined as

$$
\begin{gathered}
m=2, P_{0}=\frac{-3}{4}, P_{1}=0, P_{2}=1, F_{00}=-1, \\
\alpha_{00}=\frac{1}{2}, \beta_{00}=0, g(x)=-x^{2}+2 .
\end{gathered}
$$

We find the solution $y(x)$ with truncated Pell-Lucas series for $\mathrm{N}=2$

$$
\boldsymbol{y}_{2}(x)=\sum_{n=o}^{2} \boldsymbol{a}_{n} \boldsymbol{Q}_{n}(x)
$$

and the collocation points for $N=2 ;\left\{x_{0}=0, x_{1}=\frac{1}{2}\right.$, $\left.x_{2}=1\right\}$ are obtained. The fundamental matrix equation of the problem can be written, using Eq. (2.9), as

$$
\begin{aligned}
\left\{P_{0} X B^{0} M+P_{1} X B^{1} M+P_{2} X B^{2} M\right. \\
\left.+F_{00} X B\left(\frac{1}{2}, 0\right) B^{0} M\right\} A=G
\end{aligned}
$$

where 
$\boldsymbol{W}=\boldsymbol{P}_{0} X B^{0} M+P_{1} X B^{1} M+P_{2} X B^{2} M$

$$
+\boldsymbol{F}_{00} \boldsymbol{X} \boldsymbol{B}\left(\frac{1}{2}, 0\right) \boldsymbol{B}^{0} \boldsymbol{M} .
$$

Substituting the numerical values yields

$W=\left[\begin{array}{ccc}-3.5 & 0 & 4.5 \\ -3.5 & -1.25 & 3.5 \\ -3.5 & -2.5 & 0.5\end{array}\right]$ and $G=\left[\begin{array}{l}2 \\ 1.75 \\ 1\end{array}\right]$

The matrix forms for the initial conditions in

(2.11) are

$$
\begin{aligned}
& {\left[\mathrm{u}_{0} ; \lambda_{0}\right]=\left[\begin{array}{lllll}
2 & 0 & 2 & ; & 0
\end{array}\right]} \\
& {\left[\mathrm{u}_{1} ; \lambda_{1}\right]=\left[\begin{array}{lllll}
0 & 2 & 0 & ; & 0
\end{array}\right] .}
\end{aligned}
$$

Thus,

$\widetilde{W} A=\tilde{G} ;[\tilde{W} ; \tilde{G}]=\left[\begin{array}{ccccc}-3.5 & 0 & 4.5 & ; & 2 \\ 2 & 0 & 2 & ; & 0 \\ 0 & 2 & 0 & ; & 0\end{array}\right]$.

By solving this system, the Pell-Lucas coefficients matrix is determined as

$$
A=\left[\begin{array}{lll}
-0.25 & 0 & 0.25
\end{array}\right]^{T} .
$$

This system yields the exact solution of the problem $y(x)=x^{2}$.

Example 2. Let us now consider the first order linear differential-difference equation with variable coefficients

$$
y^{\prime \prime}(x)-y(x)+2 y(x-1)=2 e^{1-x},-1 \leq x \leq 0
$$

under the initial conditions $y(0)=1, y^{\prime}(0)=-1$. A complete solution of the problem

$y(x)=e^{-x}$. Here the coefficients of the equation are

$$
\begin{gathered}
m=2, P_{0}=-1, P_{2}=1, F_{00}=2, \\
\alpha_{00}=1, \beta_{00}=-1, g=2 e^{1-x} .
\end{gathered}
$$

We find the solution $y(x)$ with truncated Pell-Lucas series for $\mathrm{N}=2$

$$
y_{2}(x)=\sum_{n=o}^{2} a_{n} Q_{n}(x)
$$

and the collocation points for $N=2 ;\left\{x_{0}=-1, x_{1}=\right.$ $\left.\frac{-1}{2}, x_{2}=0\right\}$ are obtained. The fundamental matrix equation of the problem can be written using, Eq. (2.9), as

$$
\left\{P_{0} X B^{0}+P_{2} X B^{2}+F_{00} X B(1,-1) B^{0}\right\} M A=G
$$

where

$$
W=P_{0} X B^{0} M+P_{2} X B^{2} M F_{00} X B(1,-1) B^{0} M .
$$

Here,

$$
\boldsymbol{P}_{0}=\left[\begin{array}{ccc}
-1 & 0 & 0 \\
0 & -1 & 0 \\
0 & 0 & -1
\end{array}\right]
$$

$$
\begin{gathered}
\boldsymbol{B}(\mathbf{1},-\mathbf{1})=\left[\begin{array}{ccc}
1 & -1 & 1 \\
0 & 1 & 2 \\
0 & 0 & 1
\end{array}\right], \boldsymbol{F}_{\mathbf{0 0}}=\left[\begin{array}{lll}
2 & 0 & 0 \\
0 & 2 & 0 \\
0 & 0 & 2
\end{array}\right] \\
\boldsymbol{M}=\left[\begin{array}{lll}
2 & 0 & 2 \\
0 & 2 & 0 \\
0 & 0 & 4
\end{array}\right], \boldsymbol{X}=\left[\begin{array}{ccc}
1 & -1 & 1 \\
1 & -\frac{1}{2} & \frac{1}{4} \\
1 & 0 & 0
\end{array}\right], \\
\boldsymbol{G}=\left[\begin{array}{l}
0.27067 \\
0.44626 \\
0.73576
\end{array}\right], \boldsymbol{P}_{\mathbf{2}}=\boldsymbol{B}^{\mathbf{0}}=\left[\begin{array}{lll}
1 & 0 & 0 \\
0 & 1 & 0 \\
0 & 0 & 1
\end{array}\right] .
\end{gathered}
$$

Substituting the numerical values yields

$$
[\mathrm{W}]=\left[\begin{array}{rrr}
2 & -6 & 38 \\
2 & -5 & 27 \\
2 & -4 & 18
\end{array}\right]
$$

The matrix form for the initial condition in (2.11) is

$$
\begin{aligned}
& {\left[\mathrm{U}_{0} ; \lambda_{0}\right]=\left[\begin{array}{lllll}
2 & 0 & 2 & ; & 1
\end{array}\right]} \\
& {\left[\mathrm{U}_{1} ; \lambda_{1}\right]=\left[\begin{array}{lllll}
0 & 2 & 0 & ; & -1
\end{array}\right]}
\end{aligned}
$$

Thus, the new matrix equation based on the condition is obtained as

$\widetilde{W} A=\tilde{G}$

or

$[\tilde{W} ; \tilde{G}]=\left[\begin{array}{ccccc}2 & -6 & 38 & ; & 14.778 \\ 2 & 0 & 2 & ; & 1 \\ 0 & 2 & 0 & ; & -1\end{array}\right]$

By solving this system, the Pell-Lucas coefficients matrix is determined as

$$
A=\left[\begin{array}{lll}
0.20061 & -0.5 & 0.29939
\end{array}\right]^{T} .
$$

Thus, the approximate of the problem is obtained as 


$$
y_{2}(x) \cong 1-x+1.1976 x^{2} .
$$

Similarly, for $\mathrm{N}=3$ and $\mathrm{N}=5$, we find the following solutions:

$$
\begin{aligned}
& y_{3}(x)=1-0.99998 x+0.17341 x^{2}-0.43898 x^{3} \\
& y_{5}(x)=1-x+ 0.48844 x^{2}-0.19319 x^{3} \\
&+0.01452 x^{4}-2.0491 \times 10^{-2} x^{5}
\end{aligned}
$$

The residual errors for $\mathrm{N}=2,3$ and 5 is obtained as follows;

$$
\begin{aligned}
& \overline{R_{2}}=\int_{-1}^{0} \frac{\left|R_{2}(x)\right| d x}{|0-(-1)|}=2.7279 \times 10^{-1} \\
& \overline{R_{3}}=\int_{-1}^{0} \frac{\left|R_{3}(x)\right| d x}{|0-(-1)|}=1.7061 \times 10^{-3} \\
& \overline{R_{5}}=\int_{-1}^{0} \frac{\left|R_{5}(x)\right| d x}{|0-(-1)|}=8.42103 \times 10^{-5}
\end{aligned}
$$

Thereby, the results related with exact solution, approximate solution and residual error obtained by our method for Example 2 are demonstrated in Table 1, Figure 1 and Figure 2.

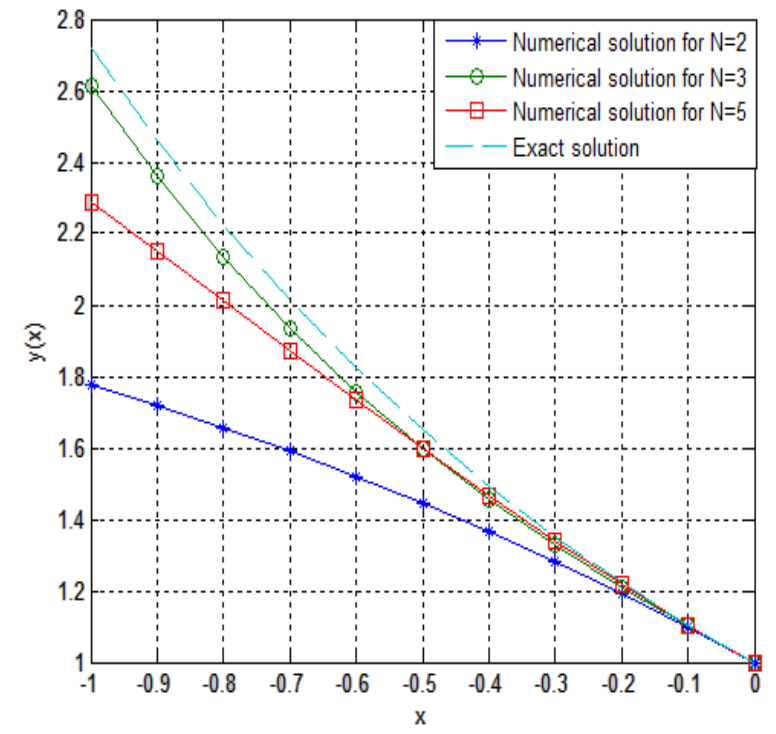

Figure 1. Exact and numerical solutions of Example 2 for $\mathrm{N}=2,3$ and 5

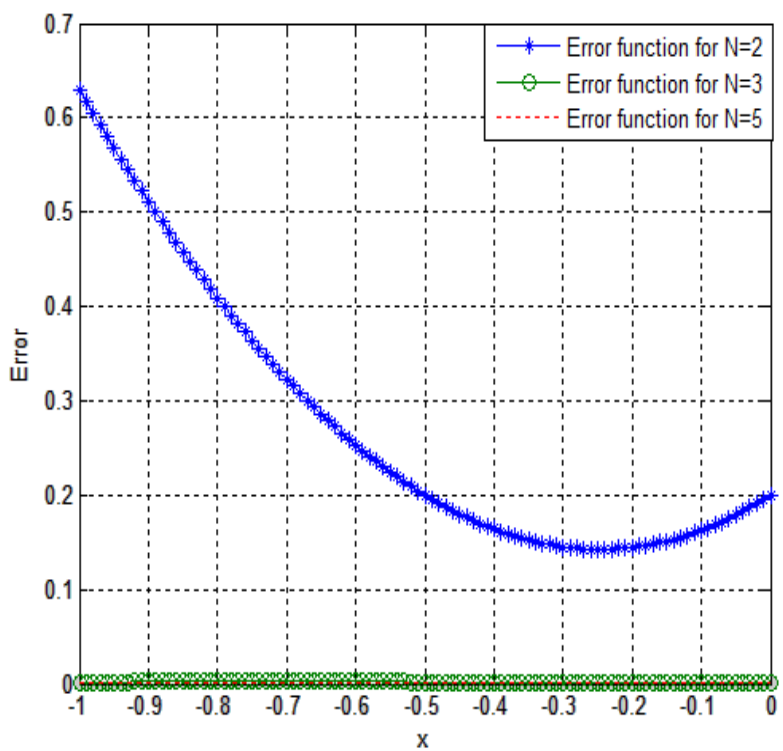

Figure 2. Residual error functions of Example 2 for $\mathrm{N}=2,3$ and 5 .

Example 3. Let us consider the third order linear delay differential equation with variable coefficients

$$
\begin{aligned}
& y^{\prime \prime \prime}(x)-x y^{\prime \prime}(x)+y^{\prime}(x)+y\left(\frac{x}{2}\right) \\
& =x \cos (2 x)+\cos \left(\frac{x}{2}\right), 0 \leq x \leq 1
\end{aligned}
$$

under the initial conditions

$$
y(0)=1, \quad y^{\prime}(0)=0, \quad y^{\prime \prime}(0)=-1 .
$$

The exact solution of the problem is $y(x)=\cos x$. Here the coefficients of the equation are

$$
\begin{gathered}
m=3, P_{0}=0, P_{1}=1, P_{2}=0, P_{3}=1, F_{00}=2, \alpha_{00} \\
=1, \beta_{00}=-1 \\
F_{20}=-x, \alpha_{20}=2, \beta_{20}=0, \\
g(x)=x \cos (2 x)+\cos \left(\frac{x}{2}\right) .
\end{gathered}
$$

Now, we look for the Pell-Lucas solution in the form

$$
y_{3}(x)=\sum_{n=0}^{3} \boldsymbol{a}_{n} \boldsymbol{Q}_{n}(x)
$$

and obtain the collocation points for

$N=3\left\{x_{0}=0, x_{1}=\frac{1}{3}, x_{2}=\frac{2}{3}, x_{3}=1\right\}$.

The fundamental matrix equation of the problem, Eq. (2.9), can be written as 


$$
\left\{\begin{array}{c}
P_{0} X B^{0}+P_{1} X B^{1}+P_{2} X B^{2}+P_{3} X B^{3} \\
+F_{00} X B_{00}\left(\frac{1}{2}, 0\right) B^{0}+F_{20} X B(2,0) B^{2}
\end{array}\right\} M A=G
$$

where

$$
\begin{aligned}
W & =P_{0} X B^{0} M+P_{1} X B^{1} M+P_{2} X B^{2} M \\
& +P_{3} X B^{3} M+F_{00} X B_{00}\left(\frac{1}{2}, 0\right) B^{0} M \\
& +F_{20} X B(2,0) B^{2} M
\end{aligned}
$$

Substituting the numerical values yields

$$
[\mathrm{W}]=\left[\begin{array}{cccc}
0 & 0 & 0 & 0 \\
2 & 7 / 3 & 19 / 9 & 1270 / 27 \\
2 & 8 / 3 & 22 / 9 & 656 / 27 \\
2 & 3 & 3 & -14
\end{array}\right] .
$$

The matrix forms for the initial conditions in are

$$
\begin{aligned}
& {\left[U_{0} ; \lambda_{0}\right]=\left[\begin{array}{llllll}
2 & 0 & 2 & 0 & ; & 1
\end{array}\right]} \\
& {\left[U_{1} ; \lambda_{1}\right]=\left[\begin{array}{llllll}
0 & 2 & 0 & 6 & ; & 0
\end{array}\right]} \\
& {\left[U_{2} ; \lambda_{2}\right]=\left[\begin{array}{llllll}
0 & 0 & 4 & 0 & ; & -1
\end{array}\right] .}
\end{aligned}
$$

Thus, the new matrix equation based on the conditions are obtained as

$$
[\tilde{W} \quad ; \quad \tilde{G}]=\left[\begin{array}{cccccc}
2 & 0 & 2 & 0 & ; & 1 \\
2 & 7 / 3 & 19 / 3 & 1270 / 27 & ; & 1.24811 \\
0 & 2 & 0 & 6 & ; & 0 \\
0 & 0 & 4 & 0 & ; & -1
\end{array}\right] .
$$

By solving this system, the Pell-Lucas coefficients matrix is determined as

$$
A=\left[\begin{array}{llll}
0.75 & -0.026722 & -0.25 & 0.006891
\end{array}\right]^{T}
$$

and the approximate the solution becomes:

$$
y_{3}(x)=1-x^{2}+5.51256 \times 10^{-2} x^{3} .
$$

Similarly we find other solutions, for $\mathrm{N}=4$ and $\mathrm{N}=5$;

$$
\begin{aligned}
y_{4}(x)=1-x^{2}+ & 7.33548 \times 10^{-2} x^{3} \\
& +3.895632 \times 10^{-3} x^{4} \\
y_{5}(x)=1-x^{2}+ & 5.6289 \times 10^{-2} x^{4}-8.7786 \times 10^{-3} x^{5} .
\end{aligned}
$$

The residual errors for $\mathrm{N}=2,3$ and 5 :

$$
\begin{aligned}
& \overline{R_{3}}=\int_{0}^{1} \frac{\left|R_{2}(x)\right| d x}{|1-0|}=9.9656 \times 10^{-2} \\
& \overline{R_{4}}=\int_{0}^{1} \frac{\left|R_{3}(x)\right| d x}{|1-0|}=1.18579 \times 10^{-2} \\
& \overline{R_{5}}=\int_{0}^{1} \frac{\left|R_{5}(x)\right| d x}{|1-0|}=2.32283 \times 10^{-2}
\end{aligned}
$$

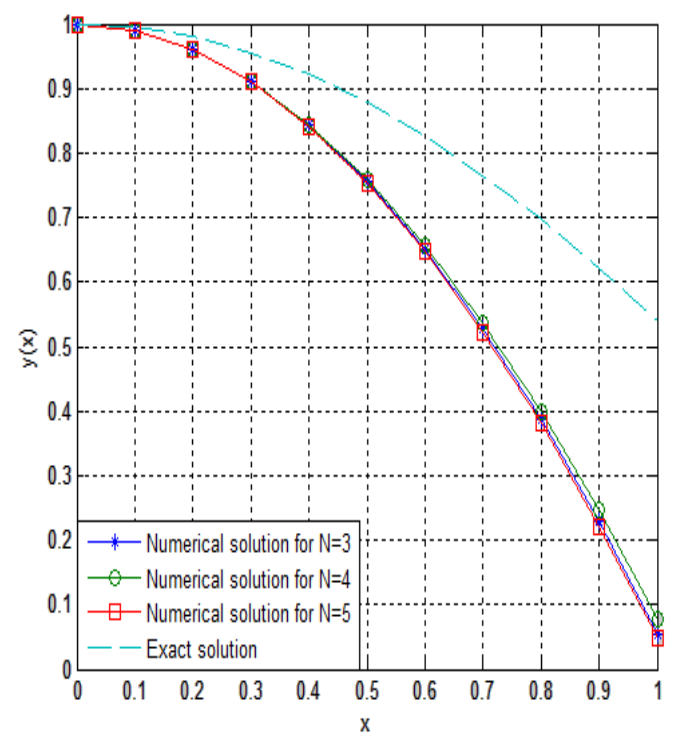

Figure 3. Comparisons of the exact and the numerical solutions of Example 3 for different $\mathrm{N}$ values

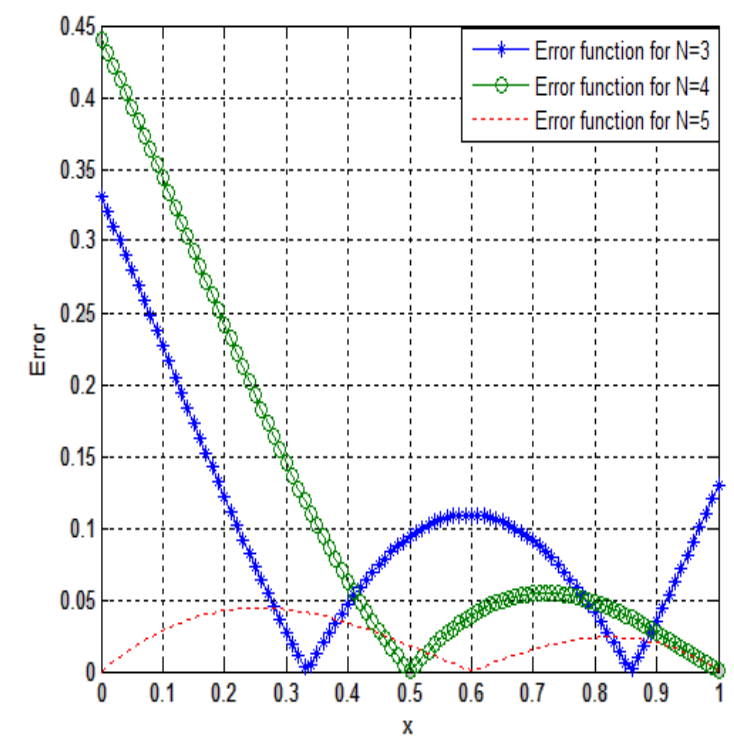

Figure 4. Residual error functions of Example 3 for $\mathrm{N}=2,3$ and 5 
Consequently, the results related with exact solution, approximate solution and residual error obtained by our method for Example 3 are demonstrated in Table 2, Figure 3 and Figure 4.

Table 1. Numerical results of the error function of Example 2 for $\mathrm{N}=2,3$ and 5

\begin{tabular}{cllclclc}
\hline $\boldsymbol{x}_{\boldsymbol{i}}$ & Exact solution & $\mathbf{N}=\mathbf{2}$ & $\left|\boldsymbol{e}_{\mathbf{2}}\left(\boldsymbol{x}_{\boldsymbol{i}}\right)\right|$ & $\mathbf{N}=\mathbf{3}$ & $\left|\boldsymbol{e}_{\mathbf{3}}\left(\boldsymbol{x}_{\boldsymbol{i}}\right)\right|$ & $\mathbf{N}=\mathbf{5}$ & $\left|\boldsymbol{e}_{\mathbf{5}}\left(\boldsymbol{x}_{\boldsymbol{i}}\right)\right|$ \\
\hline-1.0 & 2.71828 & 3.1976 & $4.73 e-01$ & 2.61237 & $1.05 e-01$ & 2.7166 & $164 e-03$ \\
-0.9 & 2.4596 & 2.87006 & $4.11 e-01$ & 2.36046 & $9.91 e-02$ & 2.4581 & $1.51 e-03$ \\
-0.8 & 2.22554 & 2.56646 & $3.41 e-01$ & 2.13572 & $8.98 e-02$ & 2.2241 & $1.35 e-03$ \\
-0.7 & 2.01375 & 2.28682 & $2.73 e-01$ & 1.93553 & $7.82 e-02$ & 2.01253 & $1.22 e-03$ \\
-0.6 & 1.82212 & 2.03114 & $2.09 e-01$ & 1.75724 & $6.48 e-02$ & 1.82104 & $1.07 e-03$ \\
-0.5 & 1.64872 & 1.7994 & $1.51 e-01$ & 1.59822 & $5.05 e-02$ & 1.64781 & $9.14 e-04$ \\
-0.4 & 1.49182 & 1.59162 & $9.97 e-02$ & 1.45583 & $3.59 e-02$ & 1.4911 & $7.28 e-04$ \\
-0.3 & 1.34986 & 1.40778 & $5.79 e-02$ & 1.32745 & $2.24 e-02$ & 1.43493 & $5.15 e-04$ \\
-0.2 & 1.22143 & 1.2479 & $2.65 e-02$ & 1.21044 & $1.09 e-02$ & 1.22111 & $2.88 e-04$ \\
-0.1 & 1.10517 & 1.11198 & $6.81 e-03$ & 1.10217 & $2.99 e-03$ & 1.10508 & $9.16 e-05$ \\
0.0 & 1.0 & 1.0 & 0 & 1.0 & 0 & 1.0 & 0 \\
\hline
\end{tabular}

Table 2. Numerical results of the error function of Example 3 for $\mathrm{N}=2,3$ and 5

\begin{tabular}{clllllll}
\hline$x_{i}$ & $\begin{array}{l}\text { Exact } \\
\text { Solution }\end{array}$ & $\mathrm{N}=3$ & $\left|e_{3}\left(x_{i}\right)\right|$ & $\mathrm{N}=4$ & $\left|e_{4}\left(x_{i}\right)\right|$ & $\mathrm{N}=5$ & $\left|e_{5}\left(x_{i}\right)\right|$ \\
\hline 0.0 & 1.0 & 1.0 & 0.0 & 1.0 & 0.0 & 1.0 & 0.0 \\
0.1 & 0.99501 & $9.91 e-01$ & $4.94 e-03$ & $9.91 e-01$ & $4.93 e-03$ & $9.91 e-01$ & $4.99 e-03$ \\
0.2 & 0.98007 & $9.60 e-01$ & $1.96 e-02$ & $9.61 e-01$ & $1.94 e-02$ & $9.60 e-01$ & $1.99 e-02$ \\
0.3 & 0.95534 & $9.11 e-01$ & $4.38 e-02$ & $9.12 e-01$ & $4.33 e-02$ & $9.10 e-01$ & $4.49 e-02$ \\
0.4 & 0.92106 & $8.43 e-01$ & $7.75 e-02$ & $8.44 e-01$ & $7.62 e-02$ & $8.41 e-01$ & $7.97 e-02$ \\
0.5 & 0.8758 & $7.56 e-01$ & $1.20 e-01$ & $7.59 e-01$ & $1.18 e-02$ & $7.53 e-01$ & $1.24 e-02$ \\
0.6 & 0.82533 & $6.51 e-01$ & $1.73 e-01$ & $6.56 e-01$ & $1.68 e-02$ & $6.46 e-01$ & $1.78 e-02$ \\
0.7 & 0.76484 & $2.89 e-01$ & $2.35 e-01$ & $5.36 e-01$ & $2.28 e-02$ & $5.22 e-01$ & $2.42 e-02$ \\
0.8 & 0.69670 & $3.88 e-01$ & $3.08 e-01$ & $3.95 e-01$ & $2.97 e-02$ & $3.80 e-01$ & $3.16 e-02$ \\
0.9 & 0.62161 & $2.31 e-01$ & $3.91 e-01$ & $2.46 e-01$ & $3.75 e-02$ & $2.21 e-01$ & $3.99 e-02$ \\
1.0 & 0.54030 & $5.51 e-02$ & $4.85 e-01$ & $7.72 e-02$ & $4.63 e-02$ & $4.75 e-02$ & $4.92 e-02$ \\
\hline
\end{tabular}

\section{Conclusion}

A new matrix method based on Pell-Lucas polynomials and collocation points is proposed to solve the highorder linear functional differential equations with hybrid delays under mixed conditions. An error analysis based on residual function is carried out to show the accuracy of the results. It is observed from the tables and figures that the error estimations are very effective. When the exact solution of the problem is not known, the error of the solution can be approximately computed by means of this residual function. In addition, we compared the numerical values of the approximate solutions obtained by the method in tables and figures. Obviously the results of the present method have been compared with the different values of N. It is also clearly seen that the Pell-Lucas matrix collocation method is more convenient to apply to linear and nonlinear integro- differential equations. However, some regularizations are required.

\section{References}

1. Reutskiy, S. Yu. A new collocation method for approximate solution of the pantograph functional differential equations with proportional delay, Applied Mathematics and Computation, 2015, 266, 642-655.

2. El-Khatib, M. A. Convergenue of the spline function for functional differential equation of neutral type, International JOurnal of Computer Mathematics. 2003, 80(11), 1437-1447.

3. Rashed, M. T. Numerical solution of functional differential, integral and integro- differential equations, Applied Mathematics and Computation, 2004, 156, 485-492.

4. Bhrawy, A. H, Assas, L. M, Tohidi, E, Alghamdi, M. A. A Legendre-Gauss collocation method for neutral functional differential equations with proportional delays, Advances in Difference Equations, 2013, (2013), 63. 
5. Heydari, M. Loghmani, G. B. Hosseini, S. M. Operational matrices of Chebyshev cardinal functions and their aplication for solving delay differential equations arising in electrodynamics with error estimation, Applied Mathematical.Modeling, 2013, 37, 7789-7809.

6. Sedaghat, S. Ordokhani, Y. Dehghan, M. Numerical solution of the delay differential equations of pantograph type via Chebyshev polynomials, Common Nonlinear Science Numerical Simulation, $2012,17,4815-4830$.

7. Akyüz, A. Sezer, A Chebyshev Collocation method for the solution of linear integro- differential equations, International Journal of Computer Mathematics, 1999, 72 (4) 491-507.

8. Gürbüz, B. Gülsu, M. Sezer, M. Numerical approach of highorder linear delay-difference equations with variable coefficients in terms of Laguerre polynomials, Mathematical and Computational Applications, 2011, 16, 267-278.

9. Wang, W. S. Li, S. F. On the one-leg Q-methods for solving nonlinear neutral functional differential equations, Applied Mathematics and Computation, 2007, 193 (1),285-301.

10. Cheng, X. Chen, Z. Zhang, Q. An approximate solution for a neutral functional- differential equation with proportional delays, Applied Mathematics and Computation, 2015, 260 27-34.

11. Kürkçü, Ö.K. Aslan, E. Sezer, M. A Novel Collocation Method Based on Residual Error Analysis for Solving Integro-Differential Equations Using Hybrid Dickson and Taylor Polynomials, Sains Malaysiana, 2017, 46, 2335-347.

12. Dai, C. Zhang, J. Jacobian elliptic function method for nonlinear differential difference equations, Chaos, Solitons \& Fractals, 2006, 27, 1042-1047.
13. Çelik, İ. Collocation method and residual correction using Chebyshev series, Applied Mathematics and Computation, 2006, 174, 910-920.

14. Wei, Y. Chen, Y. Legendre spectral collocation method for neutral and high-order Volterra integro-differential equation, Applied Numeric Mathematics, 2014, 81, 15-29.

15. Wang, K. Wang, Q. Lagrange collocation method for solving Volterra-Fredholm integral equations, Applied Mathematics and Computation, 2013, 219 10434-10440.

16. Sezer, M. Daşçığlu, A. Taylor polynomial solutions of general linear differantial-difference equations with variable coefficients. Applied Mathematics and Computation, 2006, 174, 1526-1538.

17. Sezer, M., Taylor polynomial solution of Volterra integral equations, International Journal of Mathematical Education in Science and Technology, 1994, 25, 625-633.

18. Sezer, M. and Kaynak, M. Chebyshev polynomial solutions of linear differential equations, International Journal of Mathematical Education in Science and Technology, 1996, 27, 607-618.

19. A.F.Horadam and J.M. Mahon, Pell and Pell-Lucas Polynomials, Fibonacci Quartery, 23(1), 17-20, (1985).

20. P. Filipponi, A. F. Horadam, Second derivative sequences of Fibonacci and Lucas polynomials, Fibonacci Quarterly, 31(3) (1993), 194-204. 